\title{
ENERGY ESTIMATION OF PARAMETERS OF REACTIVE POWER COMPENSATOR FOR NONLINEAR LOADS IN STEADY MODE
}

\author{
Igor Vorotnikov, Maxim Mastepanenko, Shaliko Gabrielyan, Anna Shunina \\ Stavropol State Agrarian University, Russia \\ mma_26@inbox.ru
}

\begin{abstract}
Studies on determination of reactive power, which have been conducted for more than a century, basically boil down to attempts to apply non-sinusoidal currents and voltages methods and concepts tested for the case of sinusoidal functions. The massive practical use of various semiconductor converter systems, which lead to a notable distortion of the shape of the current and voltage curves, requires a more detailed study of the problems associated with the appearance of additional energy losses and with a sharp increase of current value in the wires with a limited increase of the net power.The intensity of these processes is usually characterized by reactive power. The problem of determination and compensation of reactive power under non-linear loads becomes more typical for power supply systems of agricultural consumers. On the one hand, distorting loads, such as frequency-controlled electric drive, luminescent lighting and irradiating equipment, pulse converters and others, appear in the equipment of enterprises of the agro-industrial complex. On the other hand, the close proximity of industrial power supply networks with a high proportion of distorting loads with agricultural networks has a significant impact on the latter in the form of transformation of distorted forms of voltages and currents. The authors of this article consider the processes in the resistive branch with a key at sinusoidal electromotive force to identify the problems associated with the definition of reactive power.
\end{abstract}

Keywords: compensation, current, voltage, power, non-linear loads, power, compensation, energy.

\section{Introduction}

Modern power systems require accurate load previsions of distribution networks to achieve excellence in the operation, network planning and energy management optimization.

But most of the industrial and agricultural load is nonlinear, and the process of quality compensation is complicated due to the high level of distortion in low-voltage distribution grids. On the one hand, distorting loads, such as frequency-controlled electric drive, luminescent lighting and irradiating equipment, pulse converters and others, appear in the equipment of enterprises of the agroindustrial complex. On the other hand, the close proximity of industrial power supply networks with a high proportion of distorting loads with agricultural networks has a significant impact on the latter in the form of transformation of distorted forms of voltages and currents. The authors of this article consider the possible ways of further improvement of information-measuring and control systems compensating installations based on the analysis of already known solutions.

It should be noted that the massive practical use of various semiconductor converter systems, which lead to a notable distortion of the shape of the current and voltage curves, requires a more detailed study of the problems associated with the appearance of additional energy losses and with a sharp increase of current value in the wires with a limited increase of the net power.The intensity of these processes is usually characterized by reactive power [1].

In this field, the success depends on correct understanding of the term "reactive power", which significantly differs from the term "active power", so it is necessary to realize that the methods, which are used in the classical theory to determine the variables of electrical grids, become meaningless in the presence of nonlinear distortions in this electrical grid.

\section{Materials and methods}

The authors of this article consider the processes in the resistive branch with a key at sinusoidal electromotive force (Fig. 1) to identify the problems associated with the definition of reactive power. In this circuit at sinusoidal EMF the active resistance periodically turns on and off at periods $t_{1}<T / 2$ and $t_{2}<T / 2$. There are no reactive elements in the scheme, and for this reason the instantaneous value of power $\mathrm{p}$ is always positive, i.e. the energy flow is directed only from the source to the load (Fig. 2).

In a load, a set power, which works independently of the switching time, can be provided by changing the resistance of the resistor. And the smaller the period $\left(t_{2}-t_{1}\right)$, the lower the resistance of the resistor $r$ and the greater the instantaneous value of the current $i$. 
This irregular distribution of current during the period will be a source of additional losses, since in order to get compensation of termination of the energy flow during the current pause and the process of energy shortage replenishment current must be higher than the optimal one [2;3].

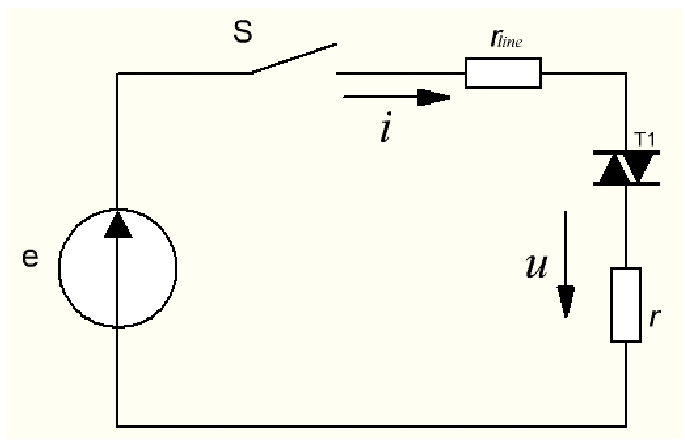

Fig. 1. Resistive branch with key at sinusoidal EMF

For the valid value of the current at:

$$
u=U_{m} \sin x \text { and } i=\frac{U_{m} \sin x}{r},
$$

where $2 \pi t_{1} / T<x<2 \pi t_{2} / T$.

$$
\begin{gathered}
U=U_{m} / \sqrt{2} \\
I_{0}=\frac{U_{m}}{r \sqrt{2}}=\frac{U}{r}
\end{gathered}
$$

where $I_{0}$ - valid value of the continuous current (in the absence of resistor switching);

$U-$ valid value of the voltage.

Determine the valid value of the intermittent current:

$$
\begin{gathered}
I=\left(1 / \pi \int_{0}^{\pi}\left(U_{m} / r\right)^{2} \sin ^{2} x d x\right)^{0.5}=U_{m} / r\left(\frac{1}{\pi}\left(\int_{0}^{\pi} \sin ^{2} x d x\right)\right)^{0,5} ; \\
I=U_{m} /(\sqrt{2} r) \sqrt{\frac{\left[2\left(x_{2}-x_{1}\right)+\left(\sin 2 x_{1}-\sin 2 x_{2}\right)\right]}{2 \pi}} \\
\alpha=\frac{\left[\left(\sin 2 x_{1}-\sin 2 x_{2}\right)+2\left(x_{2}-x_{1}\right)\right]}{2 \pi} \leq 1 \\
I=I_{0} \sqrt{\alpha}
\end{gathered}
$$

A degree of proximity of the intermittent current curve to the sine wave is characterized by the coefficient $\alpha$, if $\alpha=1$ the current curve is sinusoidal, hence the current $I$ is continuous.

For the apparent, active and reactive powers calculated from the condition of the quadratic ratio, we have:

$$
\begin{gathered}
S=U I=U I_{0} \sqrt{\alpha}, \\
P=I^{2} r=\left(I_{0} \sqrt{\alpha}\right)^{2} r=I_{0}^{2} \alpha r=I_{0} U \alpha, \\
Q^{2}=S^{2}-P^{2}=U^{2} I_{0}^{2} \alpha-I_{0}^{2} U^{2} \alpha^{2}=U^{2} I_{0}^{2}\left(\alpha-\alpha^{2}\right)=U^{2} I_{0}^{2} \alpha(1-\alpha), \\
Q=U I_{0} \sqrt{\alpha(1-\alpha)}=U I_{0} \sqrt{\alpha-\alpha^{2}}=U I_{0} \sqrt{\alpha^{2}\left(\frac{1}{\alpha}-1\right)}=U I_{0} \alpha \sqrt{\frac{1}{\alpha}-1},
\end{gathered}
$$




$$
Q=P \sqrt{(1 / \alpha-1)} .
$$

From theseequations it is clear that the reactive power calculated by the quadratic ratio differs from zero, despite the absence of reactive elements [3].

At the sinusoidal current mode, it is necessary to find the equivalent current in order to transfer the required power $\mathrm{p}$. The valid value of this equivalent current is:

$$
\tilde{I}=\frac{P}{U}=\frac{I^{2} r}{U}=\frac{\left(I_{0} \sqrt{\alpha}\right)^{2} r}{U}=\frac{I_{0}^{2} \alpha r}{U}=\frac{I_{0} \alpha I_{0} r}{U}=\frac{I_{0} \alpha U}{U}=I_{0} \alpha=\frac{I}{\sqrt{\alpha}} \alpha=I \sqrt{\alpha},
$$

that is $\sqrt{\alpha}$ less than the valid current value of the intermittent current.

It means that the energy losses are less $\alpha$ times.

Indeed, with the intermittent current at the line we have losses equal to:

$$
\Delta P_{\pi}=\left(I_{0} \sqrt{\alpha}\right)^{2} r_{\pi}=I_{0}^{2} \alpha r_{\pi} \text {. }
$$

At the sinusoidal current it is:

$$
\Delta P_{l}=(\tilde{I})^{2} r_{l}=(I \sqrt{\alpha})^{2} r_{l}=\left(I_{0} \sqrt{\alpha} \sqrt{\alpha}\right)^{2} r_{l}=I_{0}^{2} \alpha^{2} r_{l},
$$

that is $\alpha$ times less.

The resistance $\mathrm{r}$ must be set in proportion to $a$ to ensure a constant value of the power P. The amplitude of the current surge, and hence additional losses, increase with decrease of $a$ and $r$.

Figures 2 and 3 show the curves $\alpha\left(\omega t_{1}, \omega t_{2}\right)$ and $y(\alpha)=I(\alpha) / I(\widetilde{\alpha})$.

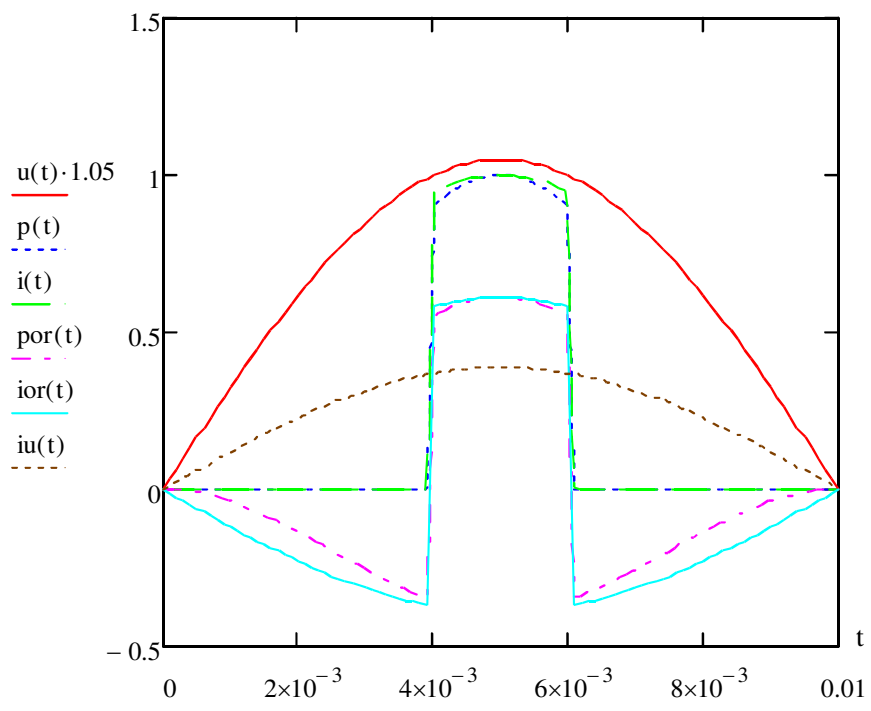

Fig. 2. Calculated curves of voltages, currents, capacities

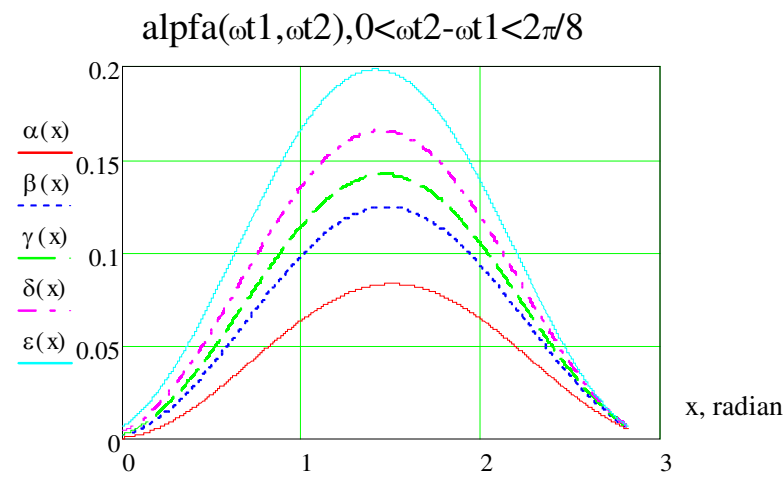

Fig. 3. Dependence of coefficient $\alpha$ on load switching of angle difference 


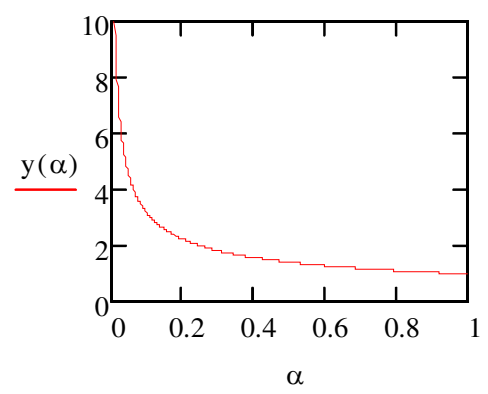

\section{Fig. 4. Dependence of ratio of valid value of intermittent current to effective value of equivalent current on coefficient $\alpha$}

These losses can be minimized only when an additional energy source with all the attributes of reactive power is added into the circuit. The additional energy source should produce a power, which average value should be zero.

Therefore, we can only talk about reactive power, which does not yet exist in the chain, but the introduction of this reactive power will minimize losses.

The average value of the energy produced by the current source for the period, when the instantaneous power of the current source is positive, i.e. for the period $\left(t_{2}-t_{1}\right)$ is equal to:

$$
\begin{gathered}
W=\frac{1}{\pi} \int_{0}^{\pi}\left(e-i_{u} r_{l}\right) i_{o r} d t \frac{1}{\pi} \int_{0}^{\pi} U_{m 1} \sin x\left(\frac{U_{m}}{r}-\frac{P}{U_{m}}\right) \sin x d x, \\
W=U_{1} I \alpha(1-\alpha) / 2=U I\left(1-\alpha r_{n} / r\right) \alpha(1-\alpha) / 2=P_{\max }\left(1-\alpha r_{n} / r\right) \alpha(1-\alpha) / 2,
\end{gathered}
$$

where $P_{\max }=U^{2} / r, U_{1}=e-I_{u} r$

$i_{u}-$ collinear (coincident to the shape of the voltage) current, $i_{u}=u P / U^{2}$;

$i_{o r}$ - orthogonal current, $i_{o r}=i-i_{u}$;

$i_{u}-$ valid value of the collinear current.

The same amount of energy this source will receive during the period of the current pause $\mathrm{T} / 2-\left(t_{2}-t_{1}\right)$.

The reactive power of the current source should be equal to $4 \mathrm{~W} / \mathrm{T}$ taking into calculation that the current source is determined by its energy. The reactive power of the current source should be equal to $4 W / T$ taking into account the actual flowing energy instead of average energy. Number 4 shows that energy, which is equal to $W$, passes through some surface; twice in one direction and twice in the opposite direction during the $T$ period. It should be noted that the expression for the collinear voltage of the current component is the main component for the decomposition approach of the time function of the current load to the components which were described just above. [4]

$$
i(t)=\frac{P}{U^{2}} u(t)=G u(t),
$$

where $G$-conductivity.

The expression (18) is known from the works of Fryze S. [5] and it is widely used by specialists.

The main disadvantage of the considered expression is the limitation of its application in the form of the requirement that the conductivity value

$$
G=P / U^{2}
$$

should be unchanged throughout the averaging time of the load power and supply voltage values during the entire period.

Therefore, there is the calculation of the correction parameters of the steady-state electromagnetic modes in the power supply system [6;7]. 
This fact introduces significant limitations on the process of reactive power compensation at nonstationary alternating nonlinear loads, which share is currently very high in power supply systems.

The authors of this article emphasized that the problem with "data types" could be solved by reconciliation "data types"; namely, integral $(P, U)$ and instantaneous $(u(t), i(t))$ characteristics of the energy regime should all be aligned.

To do this, the expression (18) is proposed to be represented as the following

$$
i_{u}(t)=u(t) \frac{P \pm \Delta p(\tau)}{(U \pm \Delta u(\tau))^{2}},
$$

where $\Delta p(\tau), \Delta u(\tau)$ are corrections of integral quantities at time $\tau<<T$. For further calculations, two observation intervals should be taken into consideration.

For certain reasons it is necessary to term them previous $(k-1)$ and current $(k)$. Both observation intervals should be equal to each other and equal to the period of the supply voltage $T$.

Taking into account the fact that the average value of periodic functions for the period depends on the position of the first (lower) limit of integration, and the time interval between the limits of integration, the following appears:

$$
\begin{gathered}
P=1 / T \int_{0}^{T} p(t) d t=1 / T \int_{\tau-T}^{\tau} p(t) d t=1 / T \int_{\tau-T}^{T} p(t) d t+1 / T \int_{T}^{\tau} p(t) d t \\
P=1 / T \int_{0}^{T} p(t) d t-1 / T \int_{0}^{\tau-T} p(t) d t+1 / T \int_{T}^{\tau} p(t) d t .
\end{gathered}
$$

The expression after taking into account the previously introduced notation for intervals of observation:

$$
P(k)=P(k-1)-1 / T \int_{0}^{\tau-T} p_{(k-1)}(t) d t+1 / T \int_{T}^{\tau} p_{(k)}(t) d t,
$$

where $\tau[T ; 2 T], \tau<<T$.

For the hardware implementation of the proposed method of averaging the periodic functions of measuring the instantaneous value of the averaged value on the $(k-1)$ interval must be in the RAM (buffer) of the computing device.

The calculated data on the $(k)$ interval are also stored in the storage device and become the data of the $(k-1)$ observation interval for $t>2 T$ time moments.

\section{Conclusions}

The proposed algorithm for calculating the integral characteristics of energy processes allows to update the data with a minimum periodicity not only for the one period of supply voltage, but for two time intervals $\tau<<T$ - for example $\tau=\{T / 4 ; T / 8 ; T / 16 ; \ldots T / 2 n\}$ where ideally $N \rightarrow \infty$.

This, in turn, leads to the expansion of the use of known control laws by compensating installations, not only in steady-state modes, but also in non-stationary and transient modes.

\section{Acknowledgements}

The research was supported by funding UDC 621.314.632.

\section{References}

[1] Zharkov F.P. About one method of determining reactive power. // Izvestiya of the Academy of Sciences of the USSR. Energy and transport. 1984. № 2. pp. 73-81.

[2] Demirchyan K.S. Reactive power for non-sinusoidal functions. Orthomality. Izvestiya of the RussianAcademy of Sciences. Power engineering. 1992. № 1, pp. 15-38. 
[3] Demirchyan K.S. Decomposition of instantaneous power into constituents. - Izvestiya of the RussianAcademy of Sciences. Power Engineering, 1994, No. 5, pp. 73-79.

[4] Asanbaev Yu. A. Fundamentals of the energy processes theory in transformation plants: Dis. ... Dr. techn. Sciences: 05.09.12: St. Petersburg, 2012. 285 c.

[5] S. Fryze. Active and Apparent power in non-sinusoidal systems. Przeglad Elektrot., No 7, 1931, pp. 93-203 (In Polish).

[6] Vorotnikov I. N., Mastepanenko, M. A., Gabrielyan Sh. Zh., Shunina A. A. Modified control algorithm for reactive power compensator for non-stationary loads. Vorotnikov // Electrical Engineering Russia, 2019, No. 3, pp. 11-14.

[7] Salmerón P., Montaño J. C., Vázquez J. R., Prieto J., Pérez A. "Compensation in Nonsinusoidal, Unbalanced Three-Phase Four-Wire Systems with Active Power Line Conditioner," IEEE Trans. Power Delivery, Vol. 19 (4), Oct. 2004, pp. 1968-1974. 